\title{
Resultados del Proyecto de Innovación y Mejora Educativa. Utilización de MATLAB como estrategia didáctica y de coordinación horizontal y vertical entre asignaturas del Grado de Ingeniería Química
}

\author{
María-Fernanda López-Pérez, S.C. Cardona, J. Lora, A. Abad, J.I. Torregrosa \\ Departamento de Ingeniería Química y Nuclear. Universitat Politècnica de València (UPV). Plaça \\ Ferràndiz i Carbonell, s/n 03801 Alcoy, Alicante (Spain). malope1@iqn.upv.es, \\ scardona@iqn.upv.es, jlora@iqn.upv.es, aabad@iqn.upv.es, jitorreg@iqn.upv.es
}

\begin{abstract}
Chemical Engineering use mathematics models in order to describe Industrial processes behavior. Nowadays, numerical software packages are a help in the acquisition of skillsin Chemical Engineering degree. In this context Matlab is software used in process simulation.

For this reason, several lectures of Escuela Politécnica Superior d'Alcoi presented an innovation and improvement educational research project (PIME) in order to used MATLAB, like coordination teaching tool between some subjects.

The principal purpose of this work is the students improvement using, as has been mentioned previously, MATLAB in a problem-based learning methodology- This methodology allows a more effective coordination in the degree. The present paper presents the PIME objectives and the first results obtained.
\end{abstract}

Keywords: Matlab, Mathematical Software like teaching tool, cross coordination

\footnotetext{
Resumen

Una de las características básicas de la Ingeniería Química es el uso de modelos matemáticos para describir el comportamiento de los procesos industriales. Por otra parte, para la adquisición adecuada de las competencias básicas que debe poseer un graduado en Ingeniería Química, en la actualidad, es necesario el uso de paquetes de software numérico de propósito general, como MATLAB, destinados a la simulación de procesos.
} 
Resultados del Proyecto de Innovación y Mejora Educativa. Utilización de MATLAB como estrategia didáctica y de coordinación horizontal y vertical entre asignaturas del Grado de Ingeniería Química

En consecuencia, varios profesores del grado dentro de un Proyecto de Innovación y Mejora Educativa (PIME) nos hemos propuesto la utilización de este software como herramienta didáctica que sirva de nexo de unión entre las asignaturas.

El principal objetivo es mejorar la coordinación entre las asignaturas del Grado de Ingeniería Química en la Escuela Politécnica Superior de Alcoy y plantear una metodología didáctica de trabajo basada en la resolución de problemas con el fin de mejorar el rendimiento académico de los alumnos.

En el siguiente trabajo se exponen los principales objetivos planteados en el proyecto de innovación y cuáles son las acciones que se están llevando a cabo para conseguirlos. También se presentarán los primeros resultados o acciones conjuntas para la mejora del Grado de Ingeniería Química.

Palabras clave: Matlab, Software como estrategia didáctica, Coordinación horizontal y vertical

\section{Introducción}

La Ingeniería Química es una rama de la Ingeniería que se dedica al estudio, síntesis, desarrollo, diseño, análisis, operación y optimización de todos aquellos procesos industriales que producen cambios físicos, químicos y/o bioquímicos en la materia. Debido a la gran cantidad de áreas y sectores en las cuales puede estar presente esta rama de la ingeniería, debe ser prioritaria y necesaria la coherencia e integración de los conceptos básicos y contenidos del Grado en Ingeniería Química, desde el primer curso hasta el Trabajo Fin de Grado.

Una de las características básicas de la Ingeniería Química es el uso de modelos matemáticos para describir el comportamiento de los procesos industriales. Este aspecto forma parte de un gran número de competencias básicas que debe adquirir un graduado en Ingeniería Química, articuladas alrededor del diseño, control y optimización de procesos o equipos. En el caso del Grado en Ingeniería Química, existen 12 competencias específicas que implican cálculo matemático de un total de 26 (VERIFICA, 2011.).

Para la adquisición adecuada de estas competencias, en la actualidad es necesario el uso de paquetes de software numérico de propósito general destinados a la simulación estacionaria y dinámica de procesos (E. Avila, 2008, C. Vazquez et al., 2003, J. Gomez, 2004). Es indudable que el uso de las herramientas informáticas de cálculo numérico, permite al alumno profundizar en el conocimiento de los procesos que estudia y afianzar las competencias adquiridas en el ámbito del cálculo y el diseño. Por una parte le facilita

(c)) EY-NC-ND 2015, Universitat Politècnica de València

Congreso IN-RED (2015) 
resolver situaciones que sería impensable hacer de una forma manual y, por otra, le permite analizar de forma sencilla el efecto que tiene la modificación de ciertas variables sobre el comportamiento de los procesos (J.F. Morales, 2013).

En el mercado existe multitud de software destinado a la resolución de modelos matemáticos, planteando por ello una gran dispersión en el uso de herramientas informáticas o software matemático que se puede utilizar para la resolución de los problemas que se plantean en las materias, que desarrollan las competencias mencionadas anteriormente.

Todo lo expuesto, nos ha llevado a una situación que se caracteriza por las siguientes dificultades en relación con la utilización del software matemático:

- El alumno puede llegar a manejar distintos software en cada asignatura, para resolver problemas en diversos ámbitos, pero que necesitan de un mismo método numérico (por ejemplo: resolver ecuaciones diferenciales ordinarias, sistemas de ecuaciones lineales, etc.).

- El alumno no llega a dominar claramente ninguno de los diversos software, debido a la limitación de tiempo que se puede dedicar en cada asignatura al aprendizaje de cada uno de ellos.

- Esta falta de coordinación entre el profesorado, en el empleo de software, provocan en el alumnado una gran incertidumbre sobre la finalidad de las herramientas matemáticas y restan tiempo para el aprendizaje de los contenidos de cada materia.

- Algunos profesores no conocen ningún software específico numérico. Por tanto, no hacen uso de ningún software matemático en sus asignaturas (utilizando simplemente calculadoras) o bien aplican un software que no es el más adecuado para los problemas concretos que se están resolviendo.

Por otra parte, es necesaria la coordinación de asignaturas en el marco del EEES. Dicha coordinación se debe abordar tanto a nivel horizontal como a nivel vertical, centrándose en coordinar las actividades, contenidos y evaluación de las competencias específicas y transversales de las asignaturas de un mismo curso o especialidad, así como asegurar la correcta distribución y consecución de las competencias específicas y transversales del título, planificando de forma adecuada la utilización de los recursos demandados [6, 7]. Esto requiere de mucha dedicación por parte de las comisiones académicas del Título y, naturalmente, del profesorado de la titulación, resultando un trabajo laborioso y complejo que debe estar muy bien planificado, por lo que será necesaria la creación de herramientas que faciliten las labores de coordinación. 
Resultados del Proyecto de Innovación y Mejora Educativa. Utilización de MATLAB como estrategia didáctica y de coordinación horizontal y vertical entre asignaturas del Grado de Ingeniería Química

\section{Objetivos}

Este trabajo tiene como principales objetivos:

1. Presentar la necesidad de realizar un proyecto coordinado entre varios profesores del Grado de Ingeniería Química utilizando MATLAB como estrategia didáctica.

2. Presentar los objetivos del proyecto de Innovación Docente y Mejora Educativa.

3. Presentar los primeros resultados de dicho proyecto.

\section{Desarrollo de la innovación}

\subsection{Antecedentes}

Durante el curso 13/14, se decidió reunir al profesorado que estuviera impartiendo docencia en el Grado de Ingeniería Química Campus de Alcoi, que utilizara programas matemáticos o hiciera uso de cálculos técnicos en sus asignaturas, y que estuviera interesado en empezar una coordinación de contenidos basándose en utilizar una metodología de resolución de problemas, que a su vez ayudara a desarrollar la competencia transversal llamada Dimensión Competencial UPV, de análisis y resolución de problemas.

Algunas conclusiones fueron:

1. Parte de los profesores que tienen asignadas asignaturas en el grado, estaban interesados en utilizar los mismos programas de cálculo para la resolución de problemas.

2. Se consideró MATLAB como el software matemático más adecuado a utilizar en el Grado en Ingeniería Química por las siguientes razones:

- Es un lenguaje de alto nivel para el cálculo numérico/simbólico empleado en el ámbito científico e ingenieril, tanto a nivel académico como industrial y en innumerables sectores.

- Es un software suficientemente contrastado que dispone de multitud de herramientas (toolboxes) especializadas.

- Constantemente se actualiza tanto el software básico como las diferentes toolboxes.

- Es utilizado por una amplia mayoría de profesores de la UPV, tanto en el ámbito docente como investigador.

- La UPV tiene la licencia de este software accesible tanto a los profesores como a los alumnos desde cualquier ordenador cuya IP ha sido dada de alta en la base de datos de la UPV. 
- Finalmente indicar que al alumno le sería fácil migrar a herramientas de cálculo numérico de uso libre y muy similares como pueden ser Scilab o Octave.

3. Esto, naturalmente, no implicaba la no utilización de otro tipo de programas matemáticos como es el caso de hojas de cálculo EXCEL (herramienta que el alumno debe conocer por su difusión en el mundo profesional) o no dar a conocer la existencia, en las asignaturas de matemáticas o informática por ejemplo, de software alternativo a MATLAB como pueda ser Mathcad o Mathematica.

4. La conveniencia de que el alumno, en las asignaturas donde necesite realizar cálculos complejos, maneje el mismo software matemático pudiendo reutilizar la programación realizada para la resolución de problemas similares en varias asignaturas del título

5. Las necesidades formativas de los docentes que no utilizaban el MATLAB y que precisarían de talleres específicos para cubrir sus necesidades en sus asignaturas.

6. La importancia de coordinar los contenidos de las diferentes asignaturas con el fin de cohesionar el título e incrementar el rendimiento académico de los alumnos.

\subsection{Descripción del trabajo}

Como se ha comentado anteriormente y detectando necesidad tanto de coordinación como de cohesión en las herramientas docentes utilizadas se presentó un PIME para el curso 2014-2015 con los objetivos de:

1. Implicar de forma activa a los profesores que tenían la inquietud e imparten asignaturas del Grado de Ingeniería Química, en la utilización de un único software matemático

2. Confeccionar un manual de utilización de la herramienta informática

3. Desarrollar una metodología de aprendizaje basada en problemas de carácter transversal que permita al alumno enlazar de forma más eficaz los contenidos más relevantes de la titulación

4. Fomentar la coordinación de los contenidos de las diferentes asignaturas dotando de una mayor cohesión a la titulación.

Las actividades que se han propuesto en el proyecto para conseguir los objetivos son:

1. Detectar las necesidades y los puntos en común respecto al cálculo matemático en las diferentes asignaturas involucradas para definir una estrategia del aprendizaje del software elegido a lo largo de los cursos del Grado.

2. Cursos de formación del profesorado implicado en consonancia con las conclusiones del apartado anterior. 
Resultados del Proyecto de Innovación y Mejora Educativa. Utilización de MATLAB como estrategia didáctica y de coordinación horizontal y vertical entre asignaturas del Grado de Ingeniería Química

3. Desarrollar curso, a curso, un método de trabajo basado en problemas-ejemplos.

4. Confeccionar un manual de referencia para alumnos y profesores que aglutine todo el trabajo realizado en el proyecto.

5. Evaluación continúa de los progresos en la coordinación horizontal y vertical de las asignaturas.

\section{Resultados}

Se han realizado reuniones de todos los profesores implicados en el título donde se han puesto de manifiesto las necesidades matemáticas de las diferentes asignaturas a cubrir por el programa MATLAB y la utilidad de ellas, es decir, específicamente "para que” fuera a ser utilizado. Para ello, se acordó elaborar una hoja de cálculo con una lista de métodos numéricos necesarios en el ámbito de la Ingeniería Química y que pueden trabajarse con MATLAB, para que cada asignatura indicara cuáles de ellos necesitaban conocer sus alumnos. En la figura 1 se muestra una parte de esta hoja de cálculo, donde las casillas verdes indican la selección realizada.

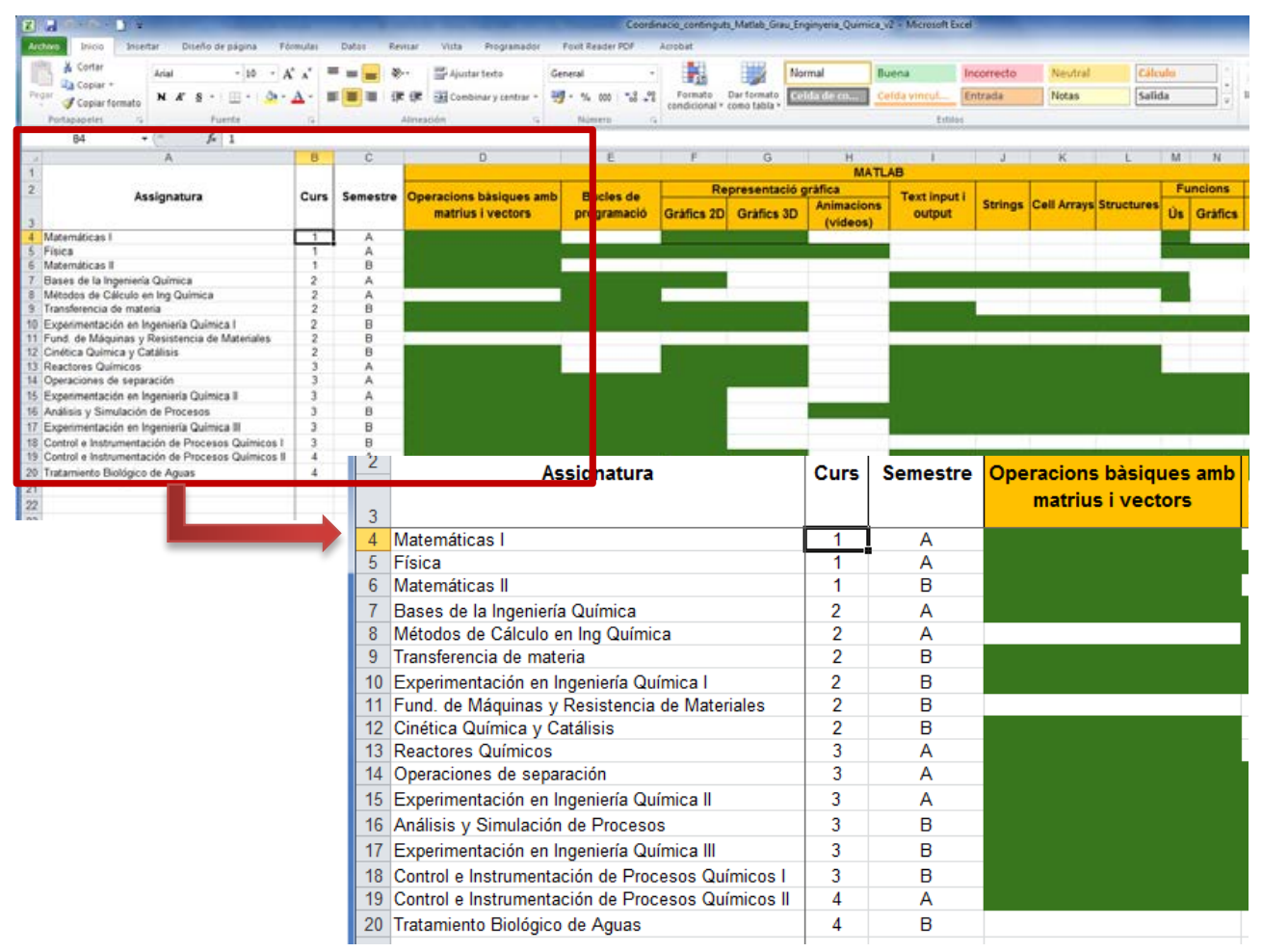

Fig. 1 Necesidades matemáticas de las diferentes asignaturas

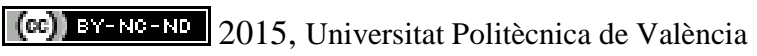

Congreso IN-RED (2015) 
Esta hoja de cálculo nos sirvió para detectar necesidades matemáticas, tanto a nivel docente como lo que los alumnos necesitan utilizar en cada una de las asignaturas. Con esta primera parte se pudo ir formando un esquema de los contenidos que deberían tener los talleres para aquellos profesores que necesitaban refuerzo. También, serviría como una guía en la realización del manual de MATLAB.

Para profundizar en los contenidos de dicho manual se pidió a los profesores implicados en el proyecto que hicieran una lista de las funciones que utilizaban de MATLAB y específicamente donde las utilizaban. Como ejemplo, vamos a ver algunas de las funciones que se utilizan en la asignatura de Física I. En la siguiente tabla se muestra un resumen de las funciones que los alumnos utilizan en la asignatura, y el objetivo que se pretende alcanzar. Con esto pretendíamos profundizar en que funciones eran las más utilizadas y la secuencia en MATLAB de aprendizaje del alumno. Con esta información se podía conocer cuáles eran las funciones que el alumno iba a utilizar, y cuales iban a ser importante recalcar o preparar en los talleres o tutoriales que se les imparte durante el grado.

Tabla 1. Ejemplos de funciones y utilizadas en MATLAB en asignaturas del Grado de Ingeniería Química

Función de Matlab

Objetivo

Asignatura

\begin{tabular}{|c|c|c|}
\hline \multirow{4}{*}{ Física I } & clc, clear, close all, disp & Comandos genéricos \\
\hline & $\begin{array}{c}\text { f eq, solve, sprint, } \\
\text { if...else...end, solve, symb, } \\
\text { pretty }\end{array}$ & $\begin{array}{l}\text { Resolución de sistemas de n } \\
\text { ecuaciones con n incógnitas para } \\
\text { resolución de problemas básicos de } \\
\text { dinámica de traslación y/o rotación } \\
\text { cuando hay varios cuerpos unidos } \\
\text { por cuerdas y por tanto varias } \\
\text { incógnitas como son la aceleración } \\
\text { del sistema y las tensiones }\end{array}$ \\
\hline & $\begin{array}{l}\text { input, roots, plot, subplot, } \\
\text { xlabel, ylabel, title, hold on, } \\
\text { text }\end{array}$ & $\begin{array}{l}\text { Representar conjuntamente la } \\
\text { trayectoria de un movimiento al } \\
\text { mismo tiempo que la variación de } \\
\text { cada coordenada con el tiempo en } \\
\text { movimientos de } 2 \text { dimensiones }\end{array}$ \\
\hline & dsolve & $\begin{array}{c}\text { Resolución de algunas ecuaciones } \\
\text { diferenciales sencillas en problemas } \\
\text { de dinámica con fuerza de fricción } \\
\text { variable }\end{array}$ \\
\hline
\end{tabular}

(c)) EY-NC-ND 2015, Universitat Politècnica de València

Congreso In-Red (2015) 
Resultados del Proyecto de Innovación y Mejora Educativa. Utilización de MATLAB como estrategia didáctica y de coordinación horizontal y vertical entre asignaturas del Grado de Ingeniería Química

También se han realizado tres talleres de MATLAB donde los docentes se van formando y adquiriendo experiencia y destreza en el manejo del proyecto. Estos seminarios han servido para poner en común problemas, deficiencias en el planteamiento inicial de la secuencia de conocimientos a adquirir por parte de los alumnos o conceptos que no se habían planteado de inicio. Se han realizado a través de ICE como curso a demanda logrando, así, un certificado de asistencia, lo cual incentiva si cabe aún más a los profesores implicados en el proyecto (Figura 2).

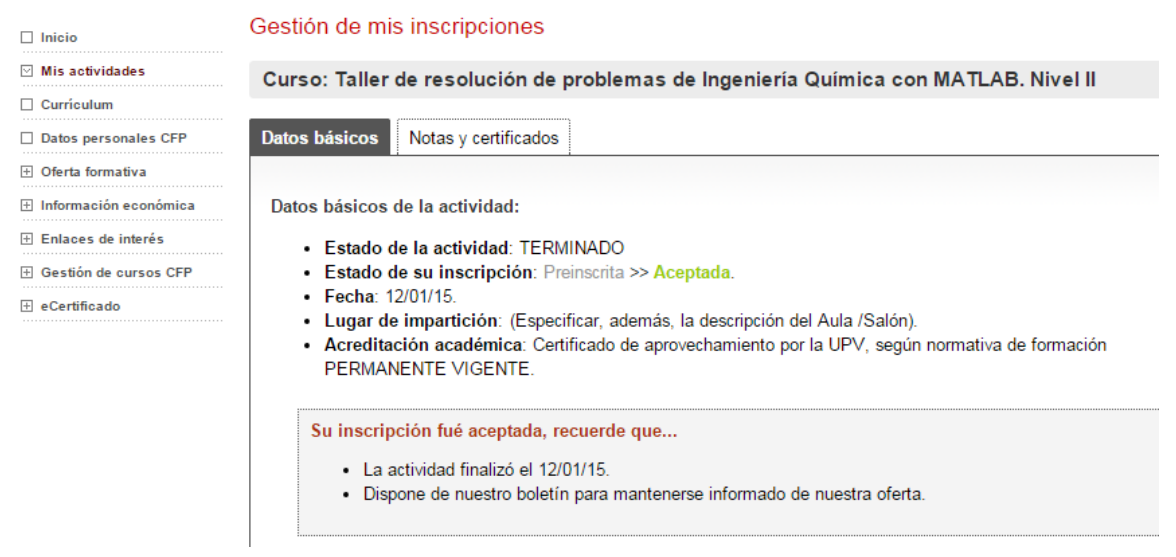

Fig. 2 Certificado Centro de Formación Permanente del taller de MATLAB.

Actualmente, se está desarrollando el primer borrador del manual de referencia con un mismo formato y varios módulos. El alumno irá recibiendo en alguna de las asignaturas del grado como Experimentación de ingeniería I, II y III seminarios donde se le entrega el módulo correspondiente a su curso y a las necesidades de cálculo. Dicho manual resumirá todas las funciones que se han visto en todas las asignaturas, utilizando ejemplos o metodologías de resolución de problemas relacionados con Ingeniería Química. Como se puede suponer, no es un documento de utilización de MATLAB, sino que será un manual de resolución de problemas de Ingeniería Química utilizando MATLAB para realizar los cálculos matemáticos. No se tratará de un manual de una asignatura determinada, sino para todo el Grado. Este manual irá actualizándose cada curso en función de las nuevas aportaciones que hagan los profesores e incluso los alumnos. La tarea de la confección del manual será realizada por todos los profesores que integran el proyecto, ofreciendo parte del contenido de sus asignaturas, sus experiencias y la metodología de resolución de los problemas que plantean en ella. El responsable del proyecto y el docente encargado de realizar los talleres de formación del profesorado, serán los principales encargados de realizar la redacción final del manual de MATLAB, ya que pueden tener una visión global de las necesidades y su secuencia temporal (Figura 3).

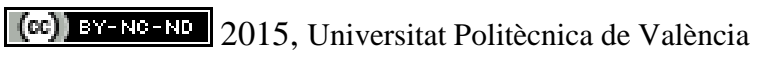


En cuanto a la evaluación se han realizado encuestas a alumnos de diferentes promociones apreciando una mejora en la percepción por parte de los alumnos de la utilidad del MATLAB en la resolución de los problemas de ingeniería química. Las encuestas se realizaron a alumnos de los tres últimos cursos, diferenciando las distintas promociones, ya que la información que puede aportar o su visión sobre las cuestiones realizadas son diferentes a medida que se va avanzando en el curso.

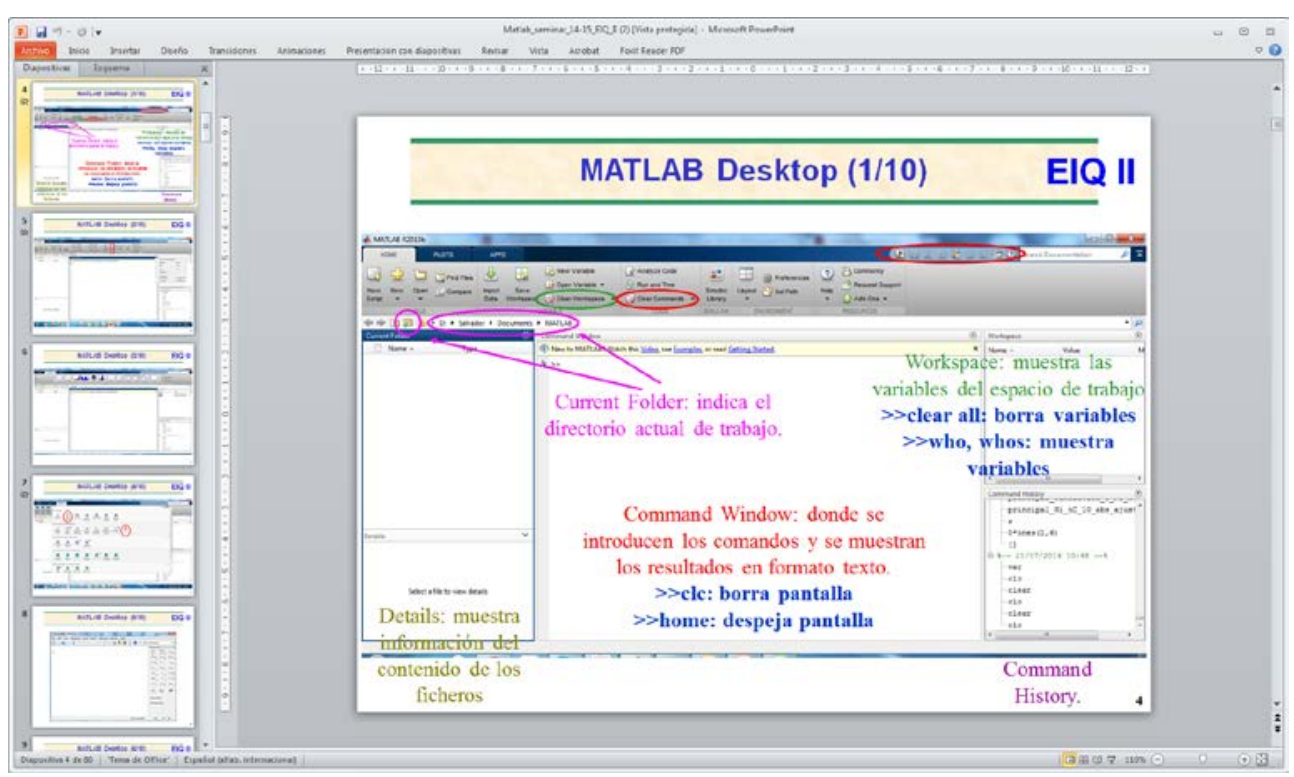

Fig. 3 Ejemplo de transparencias que formarán parte del manual de MATLAB

En la encuesta se realizaron 6 preguntas, de respuesta múltiple donde el alumno expresaba su visión de MATLAB, su aprendizaje, su uso y utilidad dentro del Grado y en su vida profesional futura. En la siguiente tabla se resume la encuesta realizada a los alumnos.

En cuanto a los resultados obtenidos en las encuestas nos indicaron que todos los alumnos consideran importante MATLAB para la resolución de problemas y que en un futuro les puede ser útil en el desarrollo de su vida profesional. En estas dos preguntas si se observa una diferencia entre los alumnos que acaban este año y los alumnos que están en segundo y tercero, ya que estos últimos consideran muy importante MATLAB en el desarrollo de la vida profesional, sujeto probablemente al aumento de la coordinación de los docentes en el uso de este software (Figura 4, respuesta 1 y 5).

También se puede observar que en la pregunta 2 (Figura 4), la percepción del nivel de conocimientos de MATLAB para la resolución de problemas se incrementa a medida que avanzan los cursos, y que los alumnos de segundo muestran su inquietud ya que es el primer curso donde se enfrentan a resolver problemas con MATLAB.

\section{(c)) EY-NC-ND 2015, Universitat Politècnica de València}


Resultados del Proyecto de Innovación y Mejora Educativa. Utilización de MATLAB como estrategia didáctica y de coordinación horizontal y vertical entre asignaturas del Grado de Ingeniería Química

Tabla 2. Encuesta del PIME (2014-2015): Utilización de Matlab como estrategia didáctica y de coordinación horizontal y vertical entre asignaturas del Grado de Ingeniería Química

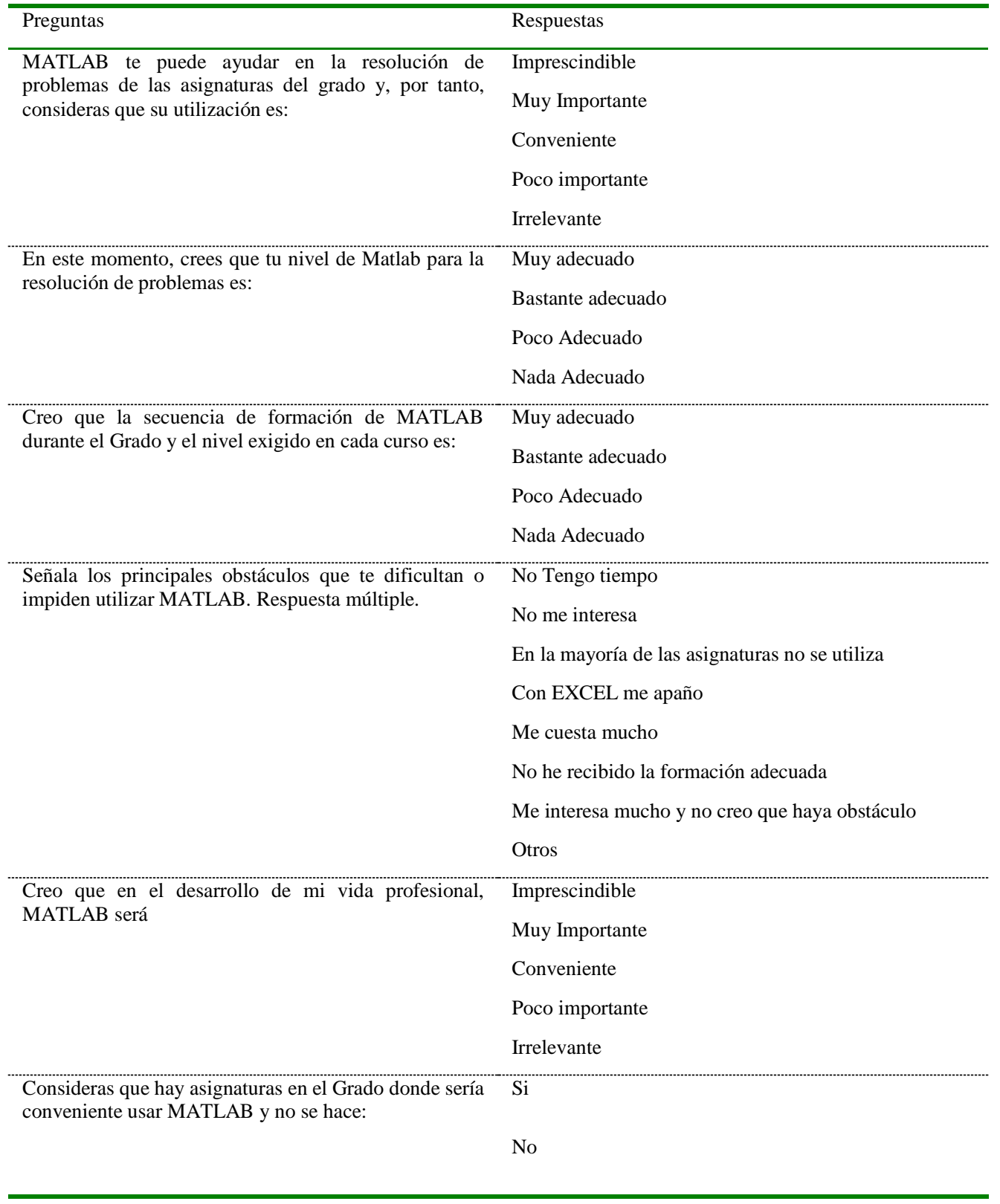

En la pregunta de cómo se está formando en el software durante el grado, se observa que los alumnos que terminan sus estudios este curso, le parece poco adecuada, pero que los 
alumnos de tercero, en los que la experiencia de los profesores era mayor y se estaba fraguando la inquietud de la coordinación de las asignaturas utilizando MATLAB han invertido la tendencia considerando que es bastante adecuada. En cuanto a los alumnos de segundo curso no tienen todavía una visión global del título y las respuestas son más dispersas (Figura 4, Respuesta 3). Cuando se analiza la respuesta 4, se observa que el mayor obstáculo de los alumnos en el uso de MATLAB es la falta de tiempo y la dificultad que tienen en el aprendizaje de MATLAB.
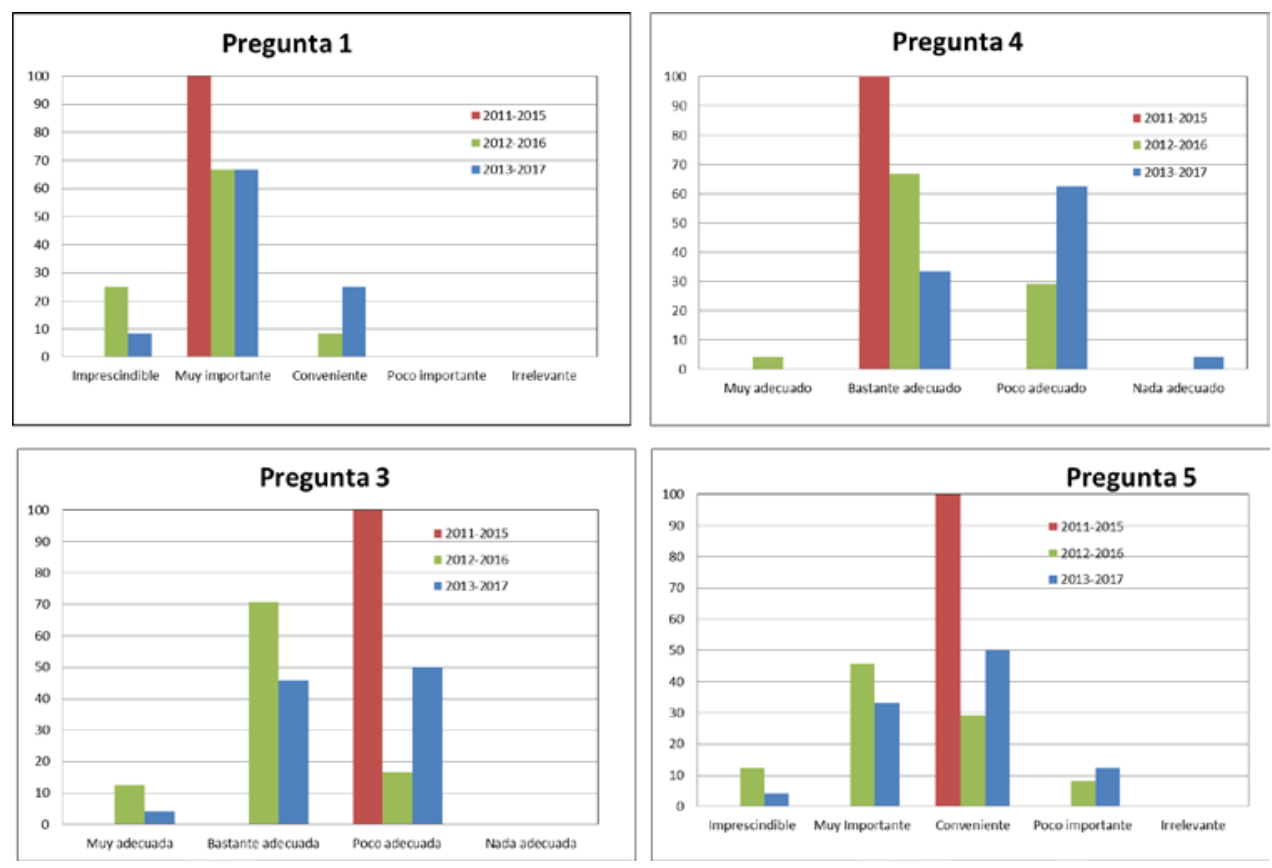

Fig. 4 Resultados de las encuestas sobre MATLAB a alumnos de 3 promociones del Grado de Ingeniería Química

\section{Conclusiones}

Los primeros resultados que se pueden extraer de este proyecto de innovación docente son prometedores, ya que la percepción de la utilidad de conocer un programa matemático en la Ingeniería Química es más positiva a medida que avanzan los cursos. Esto está permitiendo a los docentes que impartimos docencia en el Grado de Ingeniería Química una mejor coordinación en cuanto a herramientas a impartir de MATLAB y por lo tanto, un ahorro en el tiempo que se dedica dentro de cada asignatura. Para ello, el manual que se está elaborando es esencia. Naturalmente, tenemos todavía un reto importante como el presentar una coordinación vertical y horizontal real. 
Resultados del Proyecto de Innovación y Mejora Educativa. Utilización de MATLAB como estrategia didáctica y de coordinación horizontal y vertical entre asignaturas del Grado de Ingeniería Química

\section{Referencias}

E. Avila Rodríguez (2008) Experiencias en el uso del MATLAB en la enseñanza de la asignatura instrumentación y control de procesos. Innovación Tecnológica, Vol 14 (1)

IMPRESO SOLICITUD PARA VERIFICACIÓN DE TÍTULOS OFICIALES para el Grado de Ingeniería Química 2011. Documento VERIFICA.

Joan Gómez i Urgellés (2004) La ingeniería como escenario y los modelos matemáticos como actores, , XVI Simposio Iberoamericano de Enseñanza Matemática."Matemáticas para el siglo XXI"

John Fredy Morales García, Lina María Peña Páez (2013) Propuesta metodológica para la enseñanza del cálculo en ingeniería, basada en la modelación matemática., VII CIBEM

Vázquez, C., Besada, M., Le Creurer, I., García, J., Mirás, M. y Quinteiro, C. (2003) Una experiencia de utilización de internet y MatLab en la docencia de las matemáticas, Rect @, Actas 11, art. 45 (13 páginas). Recuperado el 15 de abril de 2011 de: http://www.uv.es/asepuma/XI/45.pdf 